\title{
Outbreak of Klebsiella pneumoniae carbapenemase 2-producing K. pneumoniae with high qnr prevalence in a Chinese hospital
}

\author{
Correspondence \\ Gong-Xiang Chen \\ chengong218@163.com
}

Received 6 September 2009

Accepted 10 March 2011

\author{
Rong Zhang, ${ }^{1}$ Xuan Ding Wang, ${ }^{1}$ Jia Chang Cai, ${ }^{1}$ Hong Wei Zhou, ${ }^{1}$ \\ Huo Xiang Lv, ${ }^{2}$ Oing Feng $\mathrm{Hu}^{2}$ and Gong-Xiang Chen ${ }^{1}$ \\ ${ }^{1}$ 2nd Affiliated Hospital of Zhejiang University, Zhejiang University, 88 Jiefang Road, \\ Hangzhou 310009, PR China \\ ${ }^{2}$ Zhejiang Provincial People's Hospital, 158 Shangtang Road, Hangzhou 310009, PR China
}

\section{INTRODUCTION}

Extensive use of cephalosporins has led to increased emergence of Enterobacteriaceae possessing extendedspectrum $\beta$-lactamases. Carbapenems are commonly used to treat serious infections caused by such bacteria. Carbapenem-resistant Enterobacteriaceae are uncommon in a clinical setting. However, recently, identification of carbapenem-resistant Enterobacteriaceae, especially Klebsiella pneumoniae carbapenemase (KPC)-producing bacteria, is increasing. Since the initial report of KPC-1 in K. pneumoniae in North Carolina in 2001, this enzyme has spread worldwide. The USA, Israel, South America and China are the areas where KPC-producing bacteria have been isolated most frequently (Nordmann et al., 2009). In China, a K. pneumoniae isolate from Hangzhou city producing KPC-2 was first reported in 2007 (Wei et al., 2007). In the same year, we identified KPC-2 in three Serratia marcescens isolates from the same city but a different

Abbreviations: $\mathrm{CCCP}$, carbonyl cyanide $m$-chlorophenylhydrazone; ICU, intensive care unit; KPC, Klebsiella pneumoniae carbapenemase; OMP, outer-membrane protein; $\mathrm{pl}$, isoelectric point.

Tables with the clinical details and details of the resistance genes of the isolates and the antibiotic susceptibility results are available as supplementary data with the online version of this paper. hospital (Zhang et al., 2007). Subsequently, KPC-2 was detected in a Citrobacter freundii isolate (Zhang et al., 2008), $21 \mathrm{~S}$. marcescens, $10 \mathrm{~K}$. pneumoniae, 1 Escherichia coli (Cai et al., 2008b) and 1 Enterobacter cloacae (Cai et al., 2008a).

In the present report, we describe an outbreak of 40 clinical isolates of carbapenem-resistant $K$. pneumoniae producing KPC-2, TEM, SHV and CTX-M in an intensive care unit (ICU) of another hospital in Hangzhou city, Zhejiang Provincial People's Hospital. In particular, the prevalence of the $q n r$ gene was high $(70.0 \%)$ in these KPC-2producing isolates.

\section{METHODS}

Bacterial strains. Carbapenem-resistant K. pneumoniae isolates firstly emerged in June 2007 in a surgery ICU at Zhejiang Provincial People's Hospital. In the following 7 months, a total of 40 isolates of $K$. pneumoniae with carbapenem resistance were recovered from 28 patients in the same ward. All patients had undergone surgery. All the carbapenem-resistant $K$. pneumoniae isolates during this period were collected for investigation.

Samples from pulmonary infections, wound infections, urinary tract infections and septicaemia after surgery were collected for culture. Sputum, urine and blood samples were the most common specimens. Some strains were isolated from the same patient but from a different 
clinical sample; these were also included in the investigation. Strains isolated from the same patient during the second hospitalization were also included in the investigation. Species identification was performed with the Vitek system (bioMérieux). Seventy-five per cent of patients were treated with a $\beta$-lactam/ $\beta$-lactamase inhibitor combination (including piperacillin/tazobactam and cefoperazone/ sulbactam), and about half of the patients were treated with carbapenems, quinolones or antibiotics with a strong activity against Gram-positive bacteria (including vancomycin, teicoplanin and linezolid) before the organism was isolated (within 2 weeks). Most patients received therapy with a combination of two or three kinds of antibiotics (see Supplementary Table S1 available with the online journal). K. pneumoniae $\mathrm{K} 1$ isolated at the 2nd Affiliated Hospital of Zhejiang University, China, and its E. coli transconjugant (Cai et al., 2008b) were used as control strains.

Antimicrobial-susceptibility testing. The MICs of 14 antibiotics were determined using the agar dilution method according to Clinical and Laboratory Standards Institute recommendations (CLSI, 2006).

PFGE analysis. PFGE typing of $K$. pneumoniae isolates was performed as described by PulseNet on the website of the Centers for Disease Control and Prevention (http://www.cdc.gov/pulsenet/ protocols.htm) in a Rotaphor system 6.0 instrument (Whatman Biometra). The XbaI restriction patterns of the genomic DNA of the isolates were analysed and interpreted according to the criteria described by Tenover et al. (1995).

Conjugation experiments and analysis of plasmids. Conjugation experiments were carried out in mixed broth cultures (Cai et al., 2008a). Rifampicin-resistant E. coli EC600 $\left(\mathrm{LacZ}^{-}, \mathrm{Nal}^{\mathrm{R}}, \mathrm{Rif}^{\mathrm{R}}\right)$ was used as the recipient strain. E. coli transconjugants were selected on Mueller-Hinton agar containing rifampicin $\left(500 \mu \mathrm{g} \mathrm{ml}^{-1}\right)$ and imipenem $\left(0.3 \mu \mathrm{g} \mathrm{ml}^{-1}\right)$. The colonies that grew on the selecting medium were picked up and identified using the Vitek system. Plasmids from K. pneumoniae isolates and E. coli transconjugants were extracted using an AxyPrep plasmid miniprep kit (Axygen Scientific) and examined by electrophoresis.

IEF of $\boldsymbol{\beta}$-lactamase. The crude $\beta$-lactamase extracts of $K$. pneumoniae isolates and their E. coli transconjugants were prepared by ultrasonic treatment of bacterial cells. IEF was carried out on a PhastGel polyacrylamide gel ( $\mathrm{pH} 3-9$; Amersham Biosciences) using the PhastSystem (Pharmacia Biotech) following the method of Mathew et al. (1975). $\beta$-Lactamase activity was visualized by staining the gel with $\left(500 \mu \mathrm{g} \mathrm{ml}^{-1}\right)$ nitrocefin (Oxoid). The isoelectric point (pI) was determined after comparison with those of known $\beta$ lactamases: TEM-28 (pI 6.1), SHV-7 (pI 7.6) and ACT-1 (pI 9.0).

PCR amplification and DNA sequence analysis of drugresistance genes. Plasmid DNA or genomic DNA from $K$. pneumoniae isolates and E. coli transconjugants was used as the template depending on the type of target gene in the PCR amplification. The primers used to amplify $b l_{\mathrm{TEM}}, b l a_{\mathrm{SHV}}$ and $b l a_{\mathrm{CTX}-\mathrm{M}}$ (Yu et al., 2007), plasmid-mediated AmpC genes (PérezPérez \& Hanson, 2002), plasmid-mediated quinolone-resistance genes [qnrA, qnrB and qnrS (Robicsek et al., 2006) and $a a c\left(6^{\prime}\right)$-Ib-cr (Jiang et al., 2008)], bla $a_{\mathrm{KPC}}$ (Yigit et al., 2001), other class A carbapenemases, $b l a_{\mathrm{NMC}}, b l a_{\mathrm{SME}}, b l a_{\mathrm{IMI}}$ and $b l a_{\mathrm{GES}}$, metallo- $\beta$-lactamase, $b l a_{\mathrm{IMP}-1}$, $b l a_{\mathrm{IMP}-2}, b l a_{\mathrm{VIM}-1}$ and $b l a_{\mathrm{VIM}-2}$, and a class $\mathrm{D}$ carbapenemase $b l a_{\mathrm{OXA}-48}$ (Queenan \& Bush, 2007) were as described. The reaction was conducted in a T-personal thermal cycler (Whatman Biometra). PCR amplification products were purified and sequenced directly using an ABI3730 Sequencer (Applied Biosystems). DNA sequences were compared with the reported nucleotide sequences from GenBank using the BLASTN program (http://blast.ncbi.nlm.nih.gov).
Analysis of outer-membrane proteins (OMPs). The OMPs of $K$. pneumoniae strains KP1, KP6, KP11, KP29, KP39 and K. pneumoniae ATCC 13883 were isolated as described by Hernández-Allés et al. (1999). OMP profiles were determined by SDS-PAGE using $11.6 \%$ acrylamide $/ 0.4 \%$ bisacrylamide $/ 0.1 \%$ SDS in the running gel. Samples were boiled for $5 \mathrm{~min}$ in sample buffer before electrophoresis. The $0.75 \mathrm{~mm}$ thick mini gel was run at a constant current of $20 \mathrm{~mA}$ in a Mini Protein 3 slab electrophoresis cell (Bio-Rad). Proteins were visualized by staining with Coomassie brilliant blue $\left(2.5 \mu \mathrm{g} \mathrm{ml}^{-1}\right)$.

The ompK35 and ompK36 genes of strains KP1, KP6, KP11, KP29 and KP39 were amplified by PCR (Kaczmarek et al., 2006). The products were sequenced and the sequences compared with reported sequences from GenBank database using BLASTN.

Efflux mechanism. The efflux pump activity was examined by inhibition assays with carbonyl cyanide $m$-chlorophenylhydrazone (CCCP; Sigma). The MICs of carbapenems were determined by dilution on Mueller-Hinton agar, with and without CCCP (final concentration $50 \mu \mathrm{M}$ ) (Hasdemir et al., 2004).

\section{RESULTS AND DISCUSSION}

\section{Antimicrobial susceptibility}

Forty K. pneumoniae isolates showed various levels of resistance to carbapenems. The MICs for imipenem, meropenem and ertapenem for these 40 isolates were 16 to 128,32 to $>256$ and 64 to $>256 \mu \mathrm{g} \mathrm{ml}^{-1}$, and were much higher than those of K. pneumoniae K1 (see Supplementary Table S2 available with the online journal). All of the $K$. pneumoniae isolates had high-level resistance to penicillins, cephalosporins, cefoxitin, aztreonam and quinolones, and were susceptible to aminoglycosides (except for KP25, KP33 and KP39). Interestingly, the MICs of ceftazidime were much more varied: for the majority of isolates, the MIC values were $>256 \mu \mathrm{g} \mathrm{ml}^{-1}$, while for a portion of them the MIC values were $32 \mu \mathrm{g} \mathrm{ml}^{-1}$.

\section{PFGE typing}

As shown in Fig. 1, the $40 \mathrm{~K}$. pneumoniae isolates had similar PFGE patterns. No more than five band differences were observed among the subtypes, suggesting that all isolates were closely related or possibly related to each other, and demonstrating the possibility of intra-hospital clonal dissemination of carbapenem-resistant K. pneumoniae. The PFGE patterns of 2 KPC-2-producing $K$. pneumoniae isolated from the 2nd Affiliated Hospital of Zhejiang University, C1 and C2, were quite different from those of the $40 \mathrm{~K}$. pneumoniae isolates from Zhejiang Provincial People's Hospital, indicating that they were genetically unrelated.

\section{Transfer of carbapenem resistance and plasmid analysis}

Carbapenem resistance was transferred from K. pneumoniae to E. coli EC600 after conjugation. All E. coli transconjugants exhibited significantly reduced carbapenem 


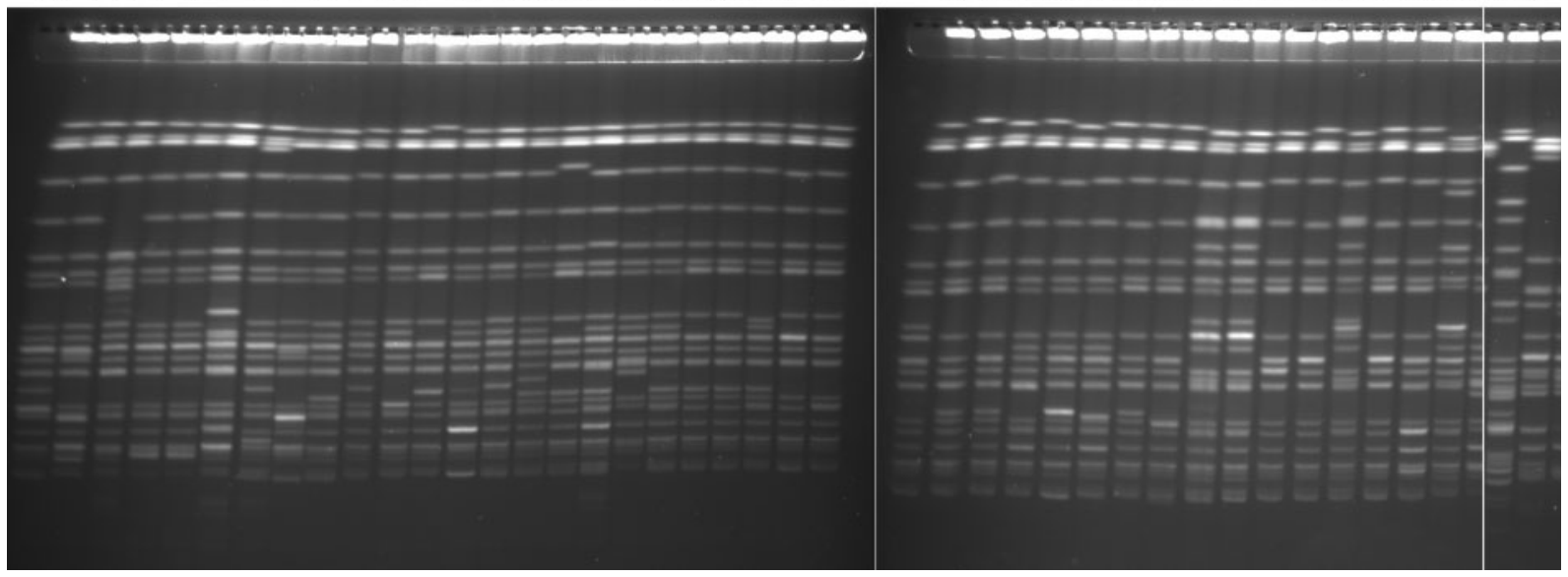

Fig. 1. PFGE patterns of chromosomal DNA restriction fragments from $K$. pneumoniae isolates. Lanes 1-40, carbapenemresistant $K$. pneumoniae KP1 to KP40, respectively; $\mathrm{C} 1$ and $\mathrm{C} 2$, two KPC-2-producing $K$. pneumoniae isolated from the 2nd Affiliated Hospital of Zhejiang University.

susceptibility. The MICs of imipenem, meropenem and ertapenem ranged from $\leqslant 0.125$ to $1-4$, from 0.5 to 4 and from 1 to $8 \mu \mathrm{g} \mathrm{ml}$, respectively. The antimicrobialsusceptibility patterns of $E$. coli transconjugants were similar to those of the donor clinical isolates of K. pneumoniae (see Supplementary Table S2 available with the online journal). They were resistant to penicillins and aztreonam, and were resistant or intermediately resistant to cefoxitin, but were susceptible to quinolones (except for the E. coli transconjugants of KP11 and KP40) and aminoglycosides. For the cephalosporins, however, the E. coli transconjugants showed various levels of resistance. The MICs of ceftazidime for 12 E. coli transconjugants were much higher than those of the other E. coli transconjugants, whilst the MICs of cefotaxime and cefepime for some E. coli transconjugants were lower than those of other transconjugants.

As shown in Fig. 2, the K. pneumoniae isolates had similar plasmid profiles. Most isolates showed five bands following electrophoresis, with sizes of approximately $55,5.6,4.2,3.9$ and $3.2 \mathrm{~kb}$, although a few isolates lacked the $3.9 \mathrm{~kb}$ band. All E. coli transconjugants acquired a plasmid of $\sim 55 \mathrm{~kb}$, and some had an additional band with a size of approximately 4.2 or $3.9 \mathrm{~kb}$.

\section{IEF analysis}

The IEF results showed that the recipient strain E. coli EC600 did not produce any $\beta$-lactamase, and had no $\mathrm{pI}$ band (data not shown). All K. pneumoniae isolates had a common band with $\beta$-lactamase activity at a $\mathrm{pI}$ of $\sim 6.7$. The majority had extra bands with pIs of 5.4 and 7.9, and a band with a pI of 7.3 was observed in some isolates. The pI 6.7 band was found uniformly in all E. coli transconjugants. To our surprise, many E. coli transconjugants also produced bands of pI 5.4, and some of them produced both 5.4 and 7.9 bands, but few had the pI 7.3 band (Fig. 3).

\section{PCR and DNA sequence analysis}

All K. pneumoniae isolates and E. coli transconjugants produced KPC-2, and most produced additional $\beta$-lactamases such as TEM-1, SHV-11/-12 and CTX-M-14/-3. A total of 39 isolates and $38 \mathrm{E}$. coli transconjugants produced TEM-1, 40 isolates produced SHV (11 were SHV-11, 29 were SHV-12, and 12 E. coli transconjugants were positive for SHV-12), 38 isolates produced CTX-M (37 were CTX$\mathrm{M}-14,1$ was CTX-M-3, and $32 \mathrm{E}$. coli transconjugants were positive for CTX-M-14), and 27 isolates carried the qnr gene (25 were qnrB2, 2 were qnrS1, and 27 E. coli transconjugants were all positive for $q n r$ ) (see Supplementary Table S1 available with the online journal). SHV-1 is chromosomally encoded in the majority of $K$. pneumoniae isolates. SHV-11 is a derivative of SHV- 1 and is a non-extended-spectrum $\beta$ lactamase. In this study, strains carrying the plasmidmediated SHV-12 should also be positive for the chromosomally mediated SHV-11. No other carbapenemase tested in this experiment was detected besides KPC. $\beta$-Lactamases with pIs of 6.7, 5.4 and 7.9 were consistent with KPC, TEM and CTX-M, respectively. The pI 7.3 band seemed to be inconsistent with SHV-11 and SHV-12 (usually pI 7.6 and 8.2 ), and the reason for this remains unclear. The level of resistance to ceftazidime for $E$. coli transconjugants was related to the genotype of SHV, as is clear from the PCR results for resistance genes and MIC values (Supplementary Tables S1 and S2 available with the online journal). Other mechanisms may contribute towards carbapenem resistance.

The MICs of the carbapenems for the K. pneumoniae isolates were significantly higher than those of the E. coli transconjugants and K. pneumoniae K1. We presumed that other carbapenem-resistance mechanisms might be involved. Therefore, analysis of outer-membrane permeability and the efflux pump activity was performed. The SDS-PAGE 


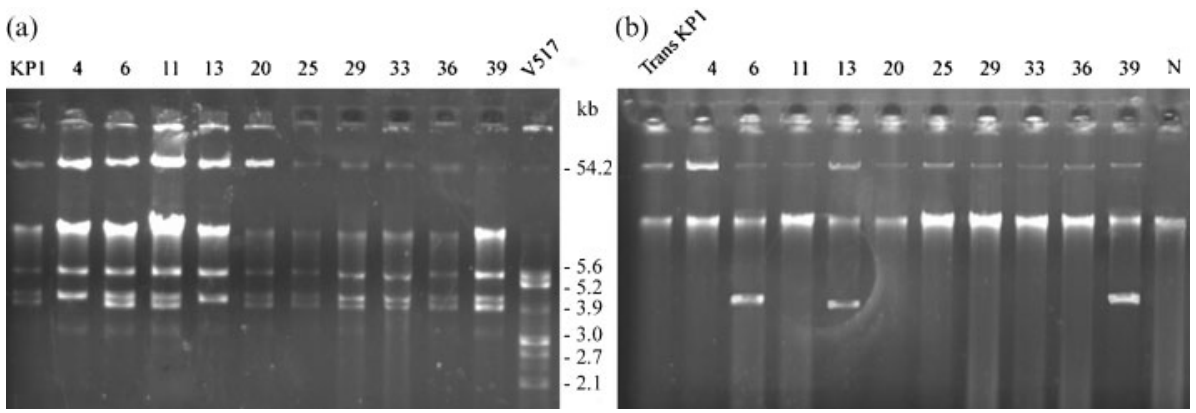

Fig. 2. Plasmid profiles of (a) partial K. pneumoniae (KP) and (b) their E. coli transconjugants (trans). Plasmids from E. coli $\mathrm{V} 517$ were used as molecular mass references. $\mathrm{N}$, negative control (E. coli EC600).

results revealed that five $K$. pneumoniae isolates did not lack OMPs. These five isolates and K. pneumoniae ATCC 13883 expressed four major OMPs, with molecular masses of approximately 49, 40, 36 and $32 \mathrm{kDa}$, which corresponded to LamB, OmpK36, OmpK35 and OmpA, respectively (Fig. 4). Amplification and sequencing of the ompK35/K36 genes indicated that the five isolates had identical gene sequences. There were a few silent point mutations in the ompK35 gene compared with that of K. pneumoniae KT755 (GenBank accession no. AJ011501), and there were several point mutations and three small DNA fragment insertions $(6,6$ and $21 \mathrm{bp}$ ) in the ompK36 gene compared with that of $K$. pneumoniae C3 (GenBank accession no. Z33506). However, the ORFs of both ompK35 and ompK36 genes were normal. The MICs of imipenem, meropenem and ertapenem with CCCP for K. pneumoniae were identical to those without CCCP, indicating that carbapenem efflux was apparently not involved in these K. pneumoniae isolates. Other mechanisms involved with the high level of resistance to carbapenems remained unclear.

\section{High prevalence of the qnr gene in KPC-2- producing $K$. pneumoniae}

KPCs are currently the most frequent class A carbapenemase and have become a matter of great concern. These enzymes have been detected in many genera and species of Enterobacteriaceae and Pseudomonas spp. in many countries (Nordmann et al., 2009). In Hangzhou city, a total of 38 KPC-2-producing bacteria were isolated from two hospitals at Zhejiang University (Cai et al., 2008a, b; Wei et al., 2007; Zhang et al., 2007, 2008). Recently, within 7 months, we isolated $40 \mathrm{KPC}-2$-producing K. pneumoniae in an ICU of another hospital in Hangzhou. These isolates showed high-level resistance to carbapenems and other $\beta$ lactams. Further studies demonstrated several resistance genes, such as TEM, SHV and CTX-M, and especially the $q n r$ gene, in these K. pneumoniae isolates. Worryingly, many of the E. coli transconjugants were positive for more than one resistance gene beside KPC-2, suggesting the coexistence of multiple resistance genes on the same plasmid, and the plasmids were able to self-transfer to other bacteria, shown at least for $E$. coli, which might cause the wide spread of multiple drug resistance. In previous studies, all $b l a_{\mathrm{KPC}}-$ encoding plasmids found in Hangzhou carried only the $b l a_{\mathrm{KPC}-2}$ gene (Cai et al., 2008a, b; Wei et al., 2007; Zhang et al., 2007, 2008). Many bla $\mathrm{KPC}^{-}$ encoding plasmids also encode $b l a_{\mathrm{TEM}-1}$ (Cuzon et al., 2008; Dortet et al., 2008; Leavitt et al., 2007; Miriagou et al., 2003; Naas et al., 2005, 2008), some encode bla $a_{\mathrm{SHV}}$ (Robicsek et al., 2006; Yigit et al., 2003), bla $a_{\mathrm{CTX}-\mathrm{M}}$ (Cuzon et al., 2008; Dortet et al., 2008; Leavitt et al.,

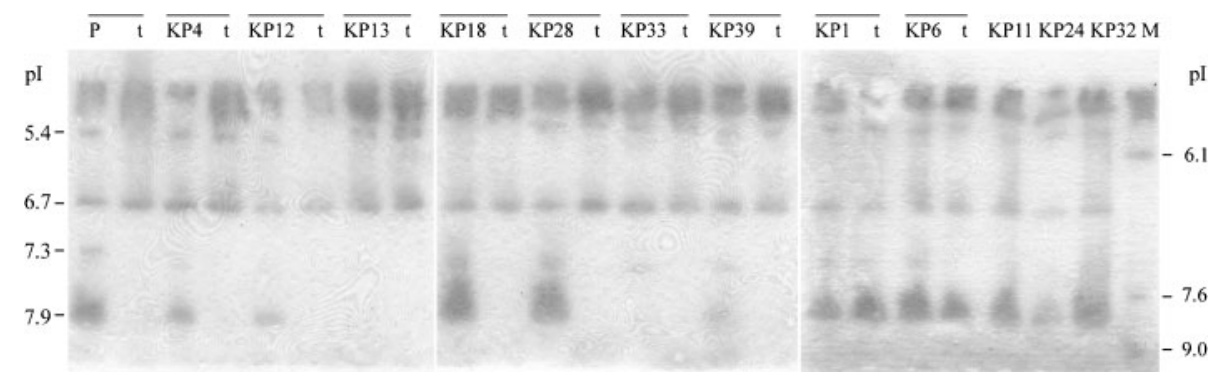

Fig. 3. IEF patterns of crude $\beta$-lactamase extracts from partial $K$. pneumoniae and their corresponding $E$. coli transconjugants (t). P, K. pneumoniae K1 isolated from the 2nd Affiliated Hospital of Zhejiang University (producing KPC-2, TEM-1, SHV-11 and CTX-M-14); t, E. coli transconjugant; M, strain producing TEM-28 (pl 6.1), SHV-7 (pl 7.6) and ACT-1 (pl 9.0). 


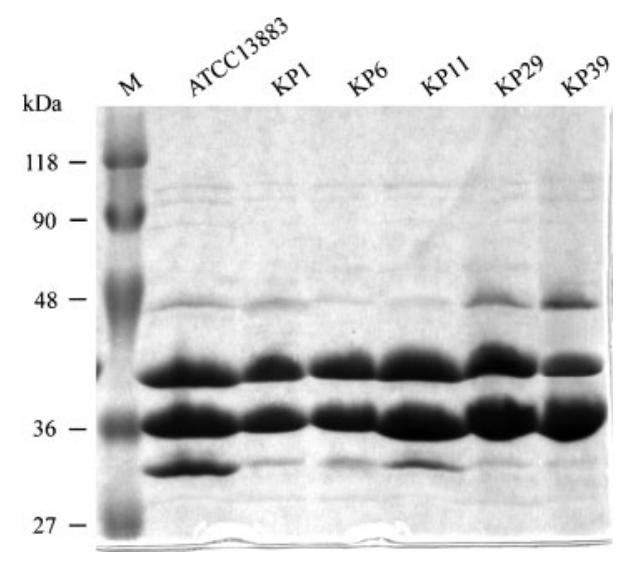

Fig. 4. SDS-PAGE analysis of OMPs from partial $K$. pneumoniae isolates and $K$. pneumoniae ATCC 13883 . M, protein molecular mass standard (MBI Fermentas).

2007; Monteiro et al., 2009; Naas et al., 2008; Tsakris et al., 2008; Yigit et al., 2003) and some encoded $q n r$ (Chmelnitsky et al., 2008; Endimiani et al., 2008; Mendes et al., 2008). Unlike these, most $b l a_{\mathrm{KPC}}$-encoding plasmids in this study were associated with four drug-resistance genes. Notably, ten of them were associated with five resistance genes, encoding KPC-2, TEM-1, SHV-12, CTXM-14 and QnrB2. Moreover, we identified qnrS associated with $b l a_{\mathrm{KPC}}$ on the same plasmid in K. pneumoniae for what is believed to be the first time. These findings warn us that novel combinations of transferable resistance determinants have emerged and could result in a serious problem regarding therapy and control. The qnrS1-positive E. coli transconjugants were intermediately resistant to ciprofloxacin, whereas the qnrB2-positive transconjugants were susceptible. Qnr determinants alone may not confer resistance to quinolones, but they can supplement other quinolone-resistance mechanisms (Martínez-Martínez et al., 2003; Poirel et al., 2006).

KPCs alone confer reduced susceptibility to carbapenems but are not sufficient to achieve full resistance, and other mechanisms, e.g. porin loss, are usually required (Nordmann et al., 2009). The K. pneumoniae isolates in this study showed various levels of resistance to carbapenems, and the MICs of two isolates (KP6 and KP29) were $>128 \mu \mathrm{g} \mathrm{ml}^{-1}$. However, the rare mechanism of carbapenem resistance in Enterobacteriaceae, the efflux pump and the common mechanism of porin deficiency were not involved. This suggests that other unknown mechanisms may contribute towards carbapenem resistance in these $K$. pneumoniae isolates, which requires further study.

\section{REFERENCES}

Cai, J. C., Zhou, H. W., Chen, G.-X. \& Zhang, R. (2008a). [Detection of plasmid-mediated carbapenem-hydrolyzing $\beta$-lactamase KPC-2 in a strain of carbapenem-resistant Enterobacter cloacae.]. Zhonghua Yi Xue Za Zhi 88, 135-138 (in Chinese).

Cai, J. C., Zhou, H. W., Zhang, R. \& Chen, G.-X. (2008b). Emergence of Serratia marcescens, Klebsiella pneumoniae, and Escherichia coli isolates possessing the plasmid-mediated carbapenem-hydrolyzing $\beta$-lactamase KPC-2 in intensive care units of a Chinese hospital. Antimicrob Agents Chemother 52, 2014-2018.

Chmelnitsky, I., Navon-Venezia, S., Strahilevitz, J. \& Carmeli, Y. (2008). Plasmid-mediated qnrB2 and carbapenemase gene bla $a_{\mathrm{KPC}-2}$ carried on the same plasmid in carbapenem-resistant ciprofloxacinsusceptible Enterobacter cloacae isolates. Antimicrob Agents Chemother 52, 2962-2965.

CLSI (2006). Methods for Dilution Antimicrobial Susceptibility Tests for Bacteria that Grow Aerobically, 7th edn, approved standard M7-A7 (M100-S16). Wayne, PA: Clinical and Laboratory Standards Institute.

Cuzon, G., Naas, T., Demachy, M. C. \& Nordmann, P. (2008). Plasmid-mediated carbapenem-hydrolyzing $\beta$-lactamase KPC-2 in Klebsiella pneumoniae isolate from Greece. Antimicrob Agents Chemother 52, 796-797.

Dortet, L., Radu, I., Gautier, V., Blot, F., Chachaty, E. \& Arlet, G. (2008). Intercontinental travels of patients and dissemination of plasmid-mediated carbapenemase KPC-3 associated with OXA-9 and TEM-1. J Antimicrob Chemother 61, 455-457.

Endimiani, A., Carias, L. L., Hujer, A. M., Bethel, C. R., Hujer, K. M., Perez, F., Hutton, R. A., Fox, W. R., Hall, G. S. \& other authors (2008). Presence of plasmid-mediated quinolone resistance in Klebsiella pneumoniae isolates possessing $b l a_{\mathrm{KPC}}$ in the United States. Antimicrob Agents Chemother 52, 2680-2682.

Hasdemir, U. O., Chevalier, J., Nordmann, P. \& Pagès, J.-M. (2004). Detection and prevalence of active drug efflux mechanism in various multidrug-resistant Klebsiella pneumoniae strains from Turkey. J Clin Microbiol 42, 2701-2706.

Hernández-Allés, S., Albertí, S., Alvarez, D., Doménech-Sánchez, A., Martínez-Martínez, L., Gil, J., Tomás, J. M. \& Benedi, V. J. (1999). Porin expression in clinical isolates of Klebsiella pneumoniae. Microbiology 145, 673-679.

Jiang, Y., Zhou, Z., Qian, Y., Wei, Z., Yu, Y., Hu, S. \& Li, L. (2008). Plasmid-mediated quinolone resistance determinants qnr and $a a c\left(6^{\prime}\right)$ $\mathrm{Ib}$-cr in extended-spectrum $\beta$-lactamase-producing Escherichia coli and Klebsiella pneumoniae in China. J Antimicrob Chemother 61, 10031006.

Kaczmarek, F. M., Dib-Hajj, F., Shang, W. \& Gootz, T. D. (2006). High-level carbapenem resistance in a Klebsiella pneumoniae clinical isolate is due to the combination of $b l a_{\mathrm{ACT}-1} \beta$-lactamase production, porin OmpK35/36 insertional inactivation, and down-regulation of the phosphate transport porin PhoE. Antimicrob Agents Chemother 50, 3396-3406.

Leavitt, A., Navon-Venezia, S., Chmelnitsky, I., Schwaber, M. J. \& Carmeli, Y. (2007). Emergence of KPC-2 and KPC-3 in carbapenemresistant Klebsiella pneumoniae strains in an Israeli hospital. Antimicrob Agents Chemother 51, 3026-3029.

Martínez-Martínez, L., Pascual, A., Garcia, I., Tran, J. \& Jacoby, G. A. (2003). Interaction of plasmid and host quinolone resistance. J Antimicrob Chemother 51, 1037-1039.

Mathew, A., Harris, A. M., Marshall, M. J. \& Ross, G. W. (1975). The use of analytical isoelectric focusing for detection and identification of $\beta$-lactamases. J Gen Microbiol 88, 169-178.

Mendes, R. E., Bell, J. M., Turnidge, J. D., Yang, Q., Yu, Y., Sun, Z. \& Jones, R. N. (2008). Carbapenem-resistant isolates of Klebsiella pneumoniae in China and detection of a conjugative plasmid (bla $a_{\mathrm{KPC}-2}$ plus qnrB4) and a bla $\mathrm{IMP}_{\mathrm{IM}}$ gene. Antimicrob Agents Chemother 52, 798-799. 
Miriagou, V., Tzouvelekis, L. S., Rossiter, S., Tzelepi, E., Angulo, F. J. \& Whichard, J. M. (2003). Imipenem resistance in a Salmonella clinical strain due to plasmid-mediated class A carbapenemase KPC-2. Antimicrob Agents Chemother 47, 1297-1300.

Monteiro, J., Santos, A. F., Asensi, M. D., Peirano, G. \& Gales, A. C. (2009). First report of KPC-2-producing Klebsiella pneumoniae strains in Brazil. Antimicrob Agents Chemother 53, 333-334.

Naas, T., Nordmann, P., Vedel, G. \& Poyart, C. (2005). Plasmidmediated carbapenem-hydrolyzing $\beta$-lactamase KPC in a Klebsiella pneumoniae isolate from France. Antimicrob Agents Chemother 49, 4423-4424.

Naas, T., Cuzon, G., Villegas, M. V., Lartigue, M. F., Quinn, J. P. \& Nordmann, P. (2008). Genetic structures at the origin of acquisition of the $\beta$-lactamase bla $a_{\mathrm{KPC}}$ gene. Antimicrob Agents Chemother 52, $1257-1263$.

Nordmann, P., Cuzon, G. \& Naas, T. (2009). The real threat of Klebsiella pneumoniae carbapenemase-producing bacteria. Lancet Infect Dis 9, 228-236.

Pérez-Pérez, F. J. \& Hanson, N. D. (2002). Detection of plasmidmediated AmpC $\beta$-lactamase genes in clinical isolates by using multiplex PCR. J Clin Microbiol 40, 2153-2162.

Poirel, L., Pitout, J. D., Calvo, L., Rodriguez-Martinez, J.-M., Church, D. \& Nordmann, P. (2006). In vivo selection of fluoroquinolone-resistant Escherichia coli isolates expressing plasmid-mediated quinolone resistance and expanded-spectrum $\beta$-lactamase. Antimicrob Agents Chemother 50, 1525-1527.

Queenan, A. M. \& Bush, K. (2007). Carbapenemases: the versatile $\beta$ lactamases. Clin Microbiol Rev 20, 440-458.

Robicsek, A., Strahilevitz, J., Sahm, D. F., Jacoby, G. A. \& Hooper, D. C. (2006). qnr Prevalence in ceftazidime-resistant Enterobacteriaceae isolates from the United States. Antimicrob Agents Chemother 50, 2872-2874.
Tenover, F. C., Arbeit, R. D., Goering, R. V., Mickelsen, P. A., Murray, B. E., Persing, D. H. \& Swaminathan, B. (1995). Interpreting chromosomal DNA restriction patterns produced by pulsed-field gel electrophoresis: criteria for bacterial strain typing. J Clin Microbiol 33, 2233-2239.

Tsakris, A., Kristo, I., Poulou, A., Markou, F., Ikonomidis, A. \& Pournaras, S. (2008). First occurrence of KPC-2-possessing Klebsiella pneumoniae in a Greek hospital and recommendation for detection with boronic acid disc tests. J Antimicrob Chemother 62, 1257-1260.

Wei, Z.-O., Du, X.-X., Yu, Y.-S., Shen, P., Chen, Y.-G. \& Li, L.-J. (2007). Plasmid-mediated KPC-2 in a Klebsiella pneumoniae isolate from China. Antimicrob Agents Chemother 51, 763-765.

Yigit, H., Queenan, A. M., Anderson, G. J., Domenech-Sanchez, A., Biddle, J. W., Steward, C. D., Alberti, S., Bush, K. \& Tenover, F. C. (2001). Novel carbapenem-hydrolyzing $\beta$-lactamase, KPC-1, from a carbapenem-resistant strain of Klebsiella pneumoniae. Antimicrob Agents Chemother 45, 1151-1161.

Yigit, H., Queenan, A. M., Rasheed, J. K., Biddle, J. W., DomenechSanchez, A., Alberti, S., Bush, K. \& Tenover, F. C. (2003). Carbapenem-resistant strain of Klebsiella oxytoca harboring carbapenem-hydrolyzing $\beta$-lactamase KPC-2. Antimicrob Agents Chemother 47, 3881-3889.

Yu, Y., Ji, S., Chen, Y., Zhou, W., Wei, Z., Li, L. \& Ma, Y. (2007). Resistance of strains producing extended-spectrum $\beta$-lactamases and genotype distribution in China. J Infect 54, 53-57.

Zhang, R., Zhou, H. W., Cai, J. C. \& Chen, G.-X. (2007). Plasmidmediated carbapenem-hydrolysing $\beta$-lactamase KPC-2 in carbapenem-resistant Serratia marcescens isolates from Hangzhou, China. J Antimicrob Chemother 59, 574-576.

Zhang, R., Yang, L., Cai, J. C., Zhou, H. W. \& Chen, G.-X. (2008). Highlevel carbapenem resistance in a Citrobacter freundii clinical isolate is due to a combination of KPC-2 production and decreased porin expression. J Med Microbiol 57, 332-337. 